\title{
Role of the Gut Microbiota in Ischemic Stroke
}

\section{(ㄷ) (1) 우) $\Theta$}

\author{
Authors \\ Katarzyna Winek1, 2, 3, Ulrich Dirnag|1, 2, 3, 4, 5, 6, Andreas Meisel1, 2, 3, 4
}

\begin{abstract}
Affiliations
1 Experimentelle Neurologie, Charité - Universitätsmedizin Berlin

2 NeuroCure Clinical Research, Charité - Universitätsmedizin Berlin

3 Centrum für Schlaganfallforschung Berlin, Charité - Universitätsmedizin Berlin

4 Klinik für Neurologie, Charité - Universitätsmedizin Berlin

5 Deutsches Zentrum für neurodegenerative Erkrankungen (DZNE), Standort Berlin

6 Deutsches Zentrum für Herz-Kreislauf-Forschung (DZHK), Standort Berlin
\end{abstract}

Key words

microbiota, microbiome, stroke, CNS

Bibliography

DOI https://doi.org/10.1055/s-0043-107843

Neurology International Open 2017; 1: E287-E293

(c) Georg Thieme Verlag KG Stuttgart · New York

ISSN 2511-1795
Correspondence

Prof. Dr. med. Andreas Meisel

Experimentelle Neurologie

Charité - Universitätsmedizin Berlin

Charitéplatz 1

10117 Berlin

Germany

andreas.meisel@charite.de

\section{ABSTRACT}

Recent studies have provided evidence for the role of the gut microbiota in the homeostasis of the central nervous system (CNS) and in the pathophysiology of its disorders, e. g. by regulation of the peripheral immune response. In this article, we discuss the importance of the gut microbiota in stroke by providing a summary of available clinical and experimental data suggesting that 1) stroke changes the gut microbiome, 2) microbiota modulates stroke outcome and 3) microbiota plays an important role in the pathogenesis of stroke (risk factors). Currently available clinical and experimental evidence suggests an important role of gut microbiota in stroke and promises clinically relevant discoveries within coming years.

\section{Introduction}

In recent years, enormous advances in molecular biology have made it possible to characterize the microorganisms that accompany the host during its lifetime. The greatest population of commensal bacteria is located in the digestive tract. More than 1000 bacterial species inhabit the intestine; most of them belonging to two phyla, Firmicutes and Bacteroidetes [1].

New sequencing and bioinformatic analysis methods have been used in studies on the role of intestinal bacteria under physiological as well as pathological conditions. The importance of intestinal microorganisms for the synthesis of some vitamins as well as the metabolism of otherwise indigestible foods has long been known [2]. Importantly, commensal bacteria support local immune system in the defense against potential pathogenic germs, and play an important role in the regulation of the systemic immune response to infectious as well as noninfectious inflammatory reactions, even those distant from the intestine [3].

Animal experiments in recent years have demonstrated that intestinal microorganisms play a very important role in the homeostasis of the central nervous system. For example, so-called germfree (GF) mice have an increased permeability of the blood-brain barrier [4], morphologically immature and functionally impaired microglia [5] and altered myelin sheaths surrounding axons in certain brain regions [6]. Associated with these are altered behavioral patterns of GF animals compared to conventionally colonized mice [7].

Nowadays the term microbiota is commonly used in the literature instead of microflora to describe all commensal microorganisms accompanying the host, since these do not belong taxonomically to the plant kingdom. The majority of commensals belong to bacteria and this group seem to be the most important physiologically. In addition, viruses, especially bacterial viruses (bacteriophages) and fungi also play an important role $[8,9]$. On the other hand, microbiome refers to the totality of all genes of the microbiota [10]. In recent years, research on the functional relevance of microbiota has developed into an important new field not only in neurosciences in general, but also in stroke research. Experimental investigations concerning changes in the microbiome after stroke as well as the influence of the microbiota on stroke prognosis have been published recently. However, robust clinical data on this topic are missing, possibly also due to problems in sample recovery in patients after an acute stroke. 
- Table 1 Rolle of intestinal microbiota in stroke, adapted from [51] with minor modifications.

\section{Stroke-related Microbiota Studies}

\section{Experimental}

\section{Clinical}

- Changes in microbiota composition in stroke animals compared to sham-operated and naïve mice; $\uparrow$ Peptococcaeae and $\downarrow$ Prevotellaceae; the changes correlate with infarct volume [12]

- $\downarrow$ Variety of species with changes in various strains; $\downarrow$ Intestinal motility after stroke; Gut as source of proinflammatory, CNS-infiltrating T-lymphocytes; Feces transplantation with balanced microbiota has neuroprotective effect [13]

- $\downarrow$ Infarct volume via reduction of IL-17 үסT cells in mice with altered microbiota [29]

- $\uparrow$ Mortality after stroke in animals after depletion of microbiota using broad-spectrum antibiotics [37]

- $\downarrow$ Stroke outcome after transplantation of microbiota from older donor animals [24]

- $\downarrow$ Neuronal damage and $\uparrow$ cognitive functions resulting from Clostridium butyricum treatment in diabetic mouse model with diminished cerebral perfusion [36]

- Translocation of bacteria from the gut after MCAo in a mouse model considered as the cause of pneumonia [25] and sepsis [23].
- Changes in the microbiome among stroke and TIA patients $\uparrow$ genera: Enterobacter, Megasphaera, Oscillibacter, Desulfovibrio; $\downarrow$ genera: Bacteroides, Prevotella, Faecalibacterium; correlating with the severity of the lesion; $\downarrow$ TMAO in stroke/TIA patients compared to patients with asymptomatic atherosclerosis [17]

- $\downarrow$ Proportion of Roseburia, Bacteroides, Faecalibacterium prausnitzii in patients after acute stroke compared to controls; $\uparrow$ Proportion of Enterobacteriaceae, Bifidobacteriaceae, Clostridium difficile among stroke patients compared to healthy volunteers; Specific changes of microbiota with restoration of microbiota composition after a few weeks [14]

- Altered composition in patients with symptomatic atherosclerosis (minor ischemic stroke, TIA) compared to healthy controls: $\uparrow$ Collinsella in atherosclerosis patients, whereas $\uparrow$ Roseburia, Eubacterium and 3 species of Bacteroides in the control group [15]

- No difference in microbiome composition between patients with symptomatic atherosclerosis (minor ischemic stroke, TIA, amaurosis fugax) and control group, various gut microorganisms identified in atherosclerotic plaques; Correlation between microbiome composition and blood lipids [16]
In this review article we will summarize the available scientific literature on the role of intestinal microbiota in ischemic stroke, while focusing on the clinically relevant aspects. We will follow 3 hypotheses which we will examine based on available data.

\section{Hypothesis 1: Ischemic stroke alters the composition of intestinal microbiota}

For virtually every previously studied disease, the exact characterization of the composition of the intestinal microbiome was the first aim in microbiological studies, in both experimental and clinical settings. As in many other areas of preclinical research, mice and rats are the most frequently used animal models in microbiome research. It should be kept in mind, however, that the results of studies in rodents cannot be directly applied to humans. Factors which differ between animals and humans, such as the physiology of the gastrointestinal tract, influence of diet or specific functions of the immune system, have different effects on the microbiota [11]. Moreover, the composition of the microbiota differs between rodents and humans per se [11].

Two experimental studies investigated changes in the composition of the microbiome after a stroke ( $\triangleright$ Table 1 ). The working group led by Denes described a relative increase of bacteria from the Peptococcaeae family (anaerobic gram-positive cocci, phylum Firmicutes, class Clostridia, order (lostridiales), with a relative reduction of bacteria of the family Prevotellaceae (anaerobic gram-negative bacilliform) in stroke mice (MCAo model; occlusion of middle cerebral artery), when compared to control animals [12]. (Refer to - Fig. 1 for the exact taxonomy of bacteria described in this article). In a similar model, the working group of Liesz observed a diminished diversity of the intestinal microbiome after stroke affecting a variety of bacterial families and genera. Here, the Bacteroidetes phylum was shown to be significantly increased [13].

Microbiome composition after cerebral ischemia has been investigated in 4 clinical trials. Using fluorescence in situ hybridization (FISH), a decreased amount of Roseburia, Bacteroides, Faecalibacterium prausnitzii and increased proportion of Enterobacteriaceae, Bifidobacteriaceae, and Clostridium difficile has been detected in gut samples of stroke patients, as compared to healthy volunteers, intensive care patients, patients with active ulcerative colitis or with irritable bowel syndrome [14]. Two sequencing studies investigated the microbiome composition of patients with atherosclerotic carotid stenosis. In one study the group of acutely symptomatic patients (TIA or mild ischemic stroke) showed differences in the intestinal microbiome [15], whereas this was not the case in the other [16].

In a larger study, stroke and TIA patients (number providing stool samples, $n=141$ ) had a significantly altered microbiome when compared to the control group (with asymptomatic atherosclerosis and without atherosclerotic changes, $n=94$ ). The composition of the intestinal microbiome also correlated with the severity of the stroke (NIHSS < 4 or $>4$ ). The microbiota of stroke and TIA patients contained significantly more bacteria of the genera Enterobacter, Megasphaera, Oscillibacter and Desulfovibrio, and less Bacteroides, Prevotella and Faecalibacterium [17].

In summary, these animal experiments and clinical data suggest that the composition of the microbiome changes after stroke. However, both the experimental and the clinical studies are not consistent regarding the respective changes, thus a characteristic "stroke profile" of altered microbiome cannot be derived from the existing data.

Systematic, standardized large-scale studies are necessary to accurately characterize alterations in the intestinal microbiota after 


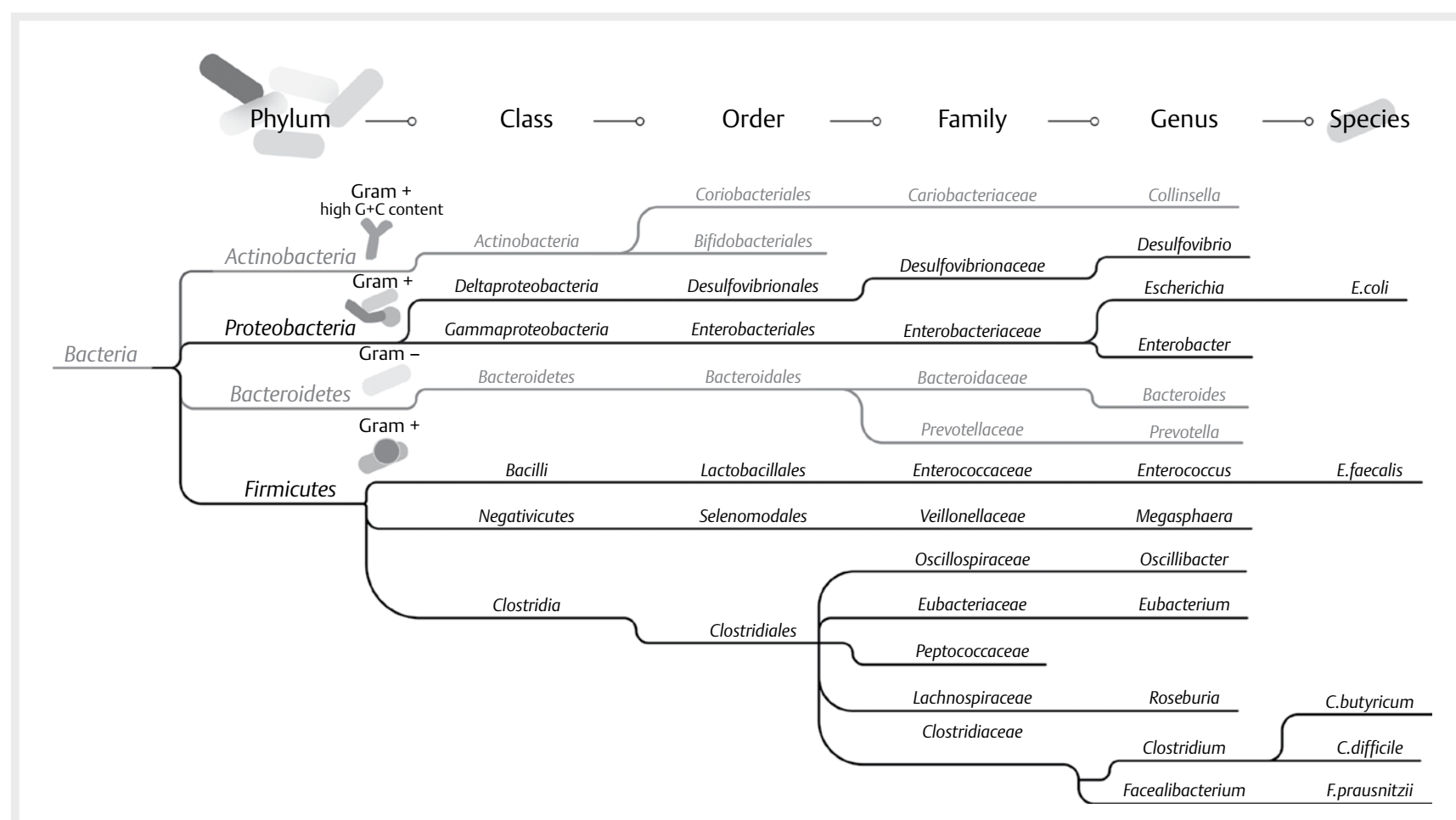

- Fig. 1 Taxonomy of microorganisms mentioned in the manuscript. More than 1000 species of bacteria inhabit the gut; more than $90 \%$ of all intestinal bacteria belong to the phylum Firmicutes or Bacteroidetes.

stroke. However, these studies will not be able to answer the therapeutically important question of whether composition of the microbiota modified before or due to the stroke has a pathophysiological significance in case of stroke.

\section{Hypothesis 2: Intestinal microbiota has an influence on cerebral ischemia or clinical progression after stroke}

The connection between brain and gut has long been known. The important role of the microbiota in this so-called gut-brain axis is being increasingly recognized [18]. Microorganisms, intestine and CNS communicate directly over the autonomic nervous system or via indirect signaling pathways through bacterial metabolic products as well as the effects of neurotransmitters on intestinal microorganisms. Interaction between intestinal microorganisms and the immune system plays a little understood, but probably very important role. Microorganisms are able to modulate the immune system. Both regulatory and pro-inflammatory effects have been described which may have consequences remote from the intestine, including in the brain [19].

An ischemic stroke can perturb the homeostasis regulated by the microbiota-gut-brain axis ( $\mathbf{F i g}$. 2). Reduced or absent intestinal motility and the disrupted intestinal barrier can lead to the translocation of intestinal bacteria into circulation and the extraintestinal organs $[12,13]$. Animal experiments also show that the local immune system of the intestine is disturbed as a result of a stroke (especially the lymphocyte population in Peyer's plaques), thus influencing the natural intestinal barrier [20]. Bacterial translocation after experimental stroke has been described, but it is neither clear whether other factors play a role in this process, nor whether this occurs in humans after stroke [21-23]. Thus, age-related degenerative processes, such as disturbed immune functions, could be a factor promoting bacterial translocation. Bacterial translocation and increased intestinal permeability was observed in both young and old mice after stroke, but had a negative effect on the stroke outcome only in old animals [23]. In older mice, translocation resulted in higher mortality due to sepsis [23]. Interestingly, the transplantation of microbiota from older animals also leads to a worsening of the prognosis after stroke [24].

The working group of Connie Wong proposed the hypothesis that translocating bacteria are the source of systemic infections, and in particular, stroke-associated pneumonia (SAP) [25]. Recent clinical and experimental studies have demonstrated that the risk of SAP is linked not only to aspiration-promoting factors such as dysphagia, but also to stroke-induced immunodepression $[26,27]$. Using complex sequencing techniques and bioinformatic models examining the microbiome in different organs, Wong and colleagues indirectly concluded that bacteria found in the lung after stroke must have entered the lung from the intestine over blood stream. The fact that germ-free (GF) mice do not develop spontaneous pneumonia after stroke, compared to specific pathogen-free (SPF) mice, is seen as proof of this. Only the oral application of Enterococcus faecalis (in GF animals) or Escherichia coli (in SPF mice) leads to pneumonia $24 \mathrm{~h}$ after experimental stroke. However, control experiments suggest that during oral application also aspiration of small quantities of bacteria is possible [25], which in stroke 


\section{immunodepression

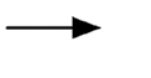 \\ systemic infection \\ bacterial translocation
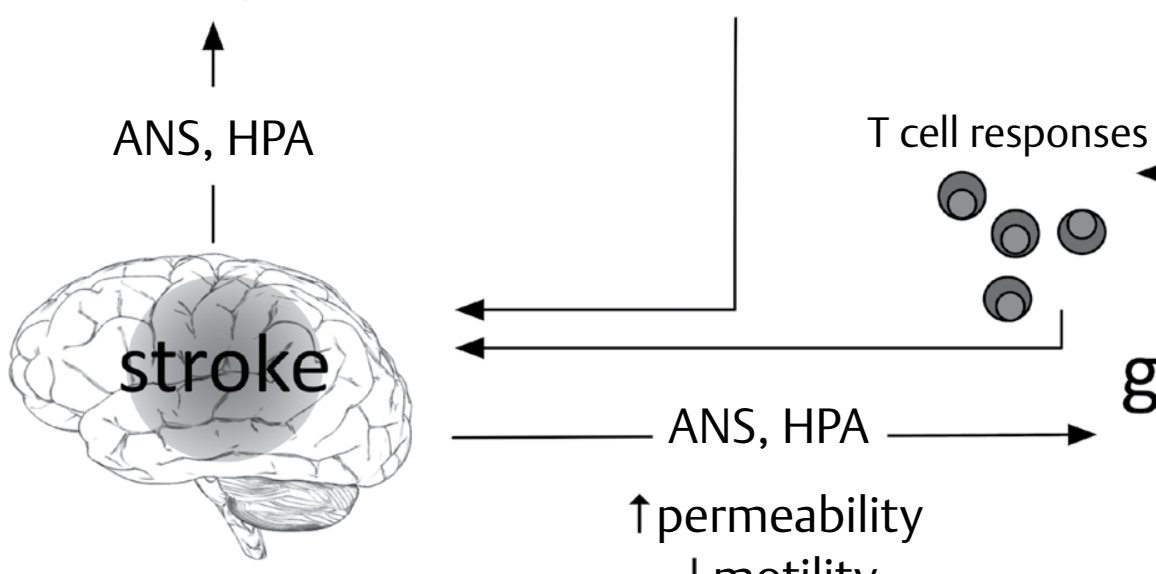 \\ $\uparrow$ permeability $\downarrow$ motility}

- Fig. 2 Brain-gut-microbiota axis after stroke. ANS: autonomic nervous system; HPA axis: hypothalamus-pituitary-adrenal cortex axis.

mice are sufficient to induce pneumonia, in contrast to control animals [28]. Consequently, the hypothesis that bacterial translocation from the intestine is the source of stroke-associated infections remains unresolved. This hypothesis is also less plausible since the spectrum of the isolated microorganisms in SAP is less attributable to the microbiota of the intestine than to the bacteria of upper respiratory tract [26].

Two experimental studies have investigated whether the composition of the microbiome has an influence on the course of stroke and examined the role of intestinal microbiota - immune interactions $[13,29]$. A short and simplified description of the immunology of stroke is presented here to allow a better understanding of these studies, after stroke activation of the autonomic nervous system leads to peripheral immunosuppression. This is caused by the apoptosis of lymphocytes and reduced production of proinflammatory cytokines such as IFNy, TNF $\alpha$ and increased production of anti-inflammatory cytokines such as IL-10 by lymphocyte subpopulations or myeloid cells, especially macrophages [27, 30]. While stroke-induced immunosuppression is widely accepted as a major contributor to the increased susceptibility to infection after acute CNS damage, it is still unclear whether this mechanism has an adaptive function that protects the ischemic brain against excessive immune reaction when the blood-brain barrier is damaged [31]. In the early post-ischemic phase the microglia become activated, followed by infiltration of peripheral immune cells, especially monocytes, $\mathrm{T}$ - and $\mathrm{B}$-lymphocytes [32, 33]. The immune reaction is also involved in regenerative processes in the CNS, with some cell populations being protective, others being damaging to brain tissue. Thus, the infiltration of Th1, Th17 and $\gamma \delta$-T cells that produce proinflammatory cytokines is linked to increased tissue damage [13, 32], whereas the anti-inflammatory regulatory T lymphocytes (Tregs) have a protective effect at least in the delayed phase after cerebral ischemia [34]. Interestingly, the intestinal microbiota is involved in the regulation of both the Th17 and Treg responses [35]. Both studies in the mouse stroke model demonstrated that the Th17- and $\mathrm{\gamma} \delta \mathrm{T}$-cells infiltrating into the CNS originate from the intestine, and the expansion of these cell types depends on the composition of the microbiota. These proinflammatory lymphocyte populations have a negative effect on stroke outcome, as measured by the larger infarct volume and poorer scores in functional tests $[13,29]$. In addition, in both experimental studies, global changes in the intestinal microbiome were induced using different approaches, in order to investigate their effects on the outcome after stroke. Both studies demonstrated that in this way not only the outcome can be improved, but that this is mediated by an enhanced Treg response [29]. In one of the two studies, it was shown that after so-called fecal microbiota transplantation, the transfer of balanced microbiota isolated from healthy mice improved the prognosis after stroke [13].

Despite all the similarities in these two experimental studies, it is not possible to conclude which subpopulations of the microbiota are protective or damaging to the ischemic brain, meaning that the "optimal" composition of the microbiota, if one can at all presume such, is still unknown. Future experimental and clinical studies will have to further characterize the complex interplay of the microbiome with the immune system in the context of stroke. The findings so far may be encouraging for the development of new therapeutic approaches, but a (targeted) manipulation of the microbiota in stroke patients cannot currently be recommended. It must be taken into account that stroke results in systemic changes affecting many organs, among others the immune system, and this would not allow a simple transfer of results of microbiota studies from healthy individuals or patients with different disease. This also applies to supposedly safe therapeutic approaches, such as probiotics [36], prebiotics, synbiotics as well as (combinations of) antibiotics. As with any other therapy, prior to instituting microbiome 
manipulations in stroke patients experimental and clinical trials should carefully investigate the effects and side effects of such an approach.

In an experimental mouse model, for example, we were able to demonstrate the fatal effect on the outcome after stroke resulting from an extensive depletion of the intestinal microbiota by the use of a broad-spectrum antibiotics. The mortality in the microbiota-depleted stroke animals was significantly increased compared to the conventionally colonized animals. This was caused by severe colitis which was fatal in the microbiota-depleted animals after stroke. This suggests that gut microbiota has a critical survival function for intestinal homeostasis in the acute course of stroke [37]. Furthermore, immunomodulatory interventions as well as microbiota manipulations, which act on the ischemic brain, can have negative effects on the immune defense against stroke-induced pneumonia. Although the proinflammatory cytokines IL-17 and TNF $\alpha$ produced by $\gamma \delta T$-cells are disadvantageous for ischemic brain parenchyma, they promote antibacterial defense by neutrophil infiltration into the infected lung tissue [38]. In addition, it must be taken into account that antibiotics as well as oral probiotics can have an effect on the microbiota of the respiratory system and thus also affect local immune response $[39,40]$. Therefore, manipulation of the intestinal microbiota should be reviewed not only for positive effects for the ischemic brain but for the whole organism as well. Experimental studies on mice can reveal basic mechanisms, as well as opportunities and risks of these therapeutic approaches. Of course, clinical trials are needed to develop successful therapies.

\section{Hypothesis 3: Intestinal microbiota plays a role in the pathogenesis of stroke}

Microorganisms have repeatedly been causally associated with atherosclerosis, one of the most important risk factors for stroke. Pathogens such as Chlamydia pneumoniae, Mycoplasma pneumoniae, Helicobacter pylori or Porphyromonas gingivalis have been associated with atherosclerosis and cardiovascular diseases [41]. In recent years, the influence of intestinal microbiome on atherosclerosis has been the focus of clinical studies. Koren et al. compared the composition of gut microbiota of healthy individuals with patients with symptomatic atherosclerosis. Although no differences in the microbiome between both groups were detectable, DNA from microorganisms of the intestinal microbiota could be identified in atherosclerotic plaques [16].

The microbial metabolite trimethylamine appears to play an important role in atherogenesis. Oxidized trimethylamine (TMAO) induces the formation of foam cells as well as hyperactivity of platelets resulting in the formation of atherosclerotic plaques $[42,43]$. Mouse experiments [44] as well as clinical trials [42, 44, 45] have shown that an increased plasma concentration of TMAO correlates with the development of atherosclerotic plaque and resulting cardiovascular events. In contrast is the observation that atherosclerotic plaques are more pronounced in the animal model of atherosclerosis in the absence of microorganisms (germ-free ApoE knockout mice) than in conventionally-colonized mice $[46,47]$. Likewise contradictory is the fact that elevated TMAO concentrations are related to a higher risk for heart failure [48], but a lower cardiovascular risk after bariatric surgery [49]. Moreover, in the aforementioned study by Yin et al., stroke and TIA patients had lower TMAO plasma concentrations compared to control group patients with asymptomatic atherosclerosis [17].

In addition to atherosclerosis, there are also other risk factors for stroke [50], such as arterial hypertension, diabetes and obesity, for which clinical and experimental data indicate that gut microbiota is related to their pathogenesis [51]. However, based to the existing data, the relationship cannot be ascertained. Additionally, it cannot be stated with certainty which bacterial subpopulations of the intestinal microbiota have positive or negative effects on the direct or indirect pathogenesis of stroke.

\section{The problem of multiple factors influenc- ing the intestinal microbiome in clinical stroke research}

Recent years resulted in generation of substantial data regarding the relationship of microbiota and pathological processes of brain disease. However, only a few animal experiments and clinical studies are available for stroke. The available study data are often contradictory in their results and conclusions. Independently reproduced results are the exception.

In the clinical setting, the composition of the microbiota can be influenced by a number of factors that are difficult to control. Thus the gut microbiota is influenced by diet, genetic factors, lifestyle, stress, age and comorbidities [52]. In particular, diet usually changes after an acute stroke, such as due to hospital food or, in the case of dysphagia, tube feeding. The effects of stroke on the gastrointestinal tract itself are well known based on clinical practice. Acute stroke often reduces intestinal motility, sometimes leading to severe constipation. More rare are serious complications, such as gastrointestinal hemorrhage [53]. Factors such as altered intestinal motility alone can affect the intestinal microbiota [54]. An additional factor complicating the interpretation and performance of clinical microbiome research in stroke patients is the relatively frequent treatment with antibiotics due to stroke-associated infections. Frequently broad-spectrum antibiotics are administered which have substantial effect on gut microbiota. Clinical and experimental studies have demonstrated that this type of antibiotic treatment alter the composition of intestinal microbiota over a long period [55]. Clinically and pragmatically, an effective anti-infective therapy with antibiotics is often indicated, but the effects of such therapies on the composition and function of the intestinal microbiota have not yet been investigated in stroke patients.

Although the extent of the influencing factors is lower or better controllable in the experimental setting, international standards are missing for experimental microbiome research. Standardization of sampling, DNA extraction and sequencing protocols as well as bioinformatic analysis are necessary for the comparability and reproducibility of the study results [56]. These problems are not specific to clinical and experimental microbiome research in the stroke field, but are found in most areas of biomedical research.

Moreover, microbiota issues have further implications. The composition of the (intestinal) microbiome is a previously unknown or not considered variable in stroke research which can "uncontrolla- 
bly" affect study results [57-59]. If intestinal microorganisms play such an important role in host organism homoeostasis, as the studies so far suggest, then systematic characterization of the microbiome would have to be included in future clinical and experimental studies [58].

\section{Summary}

Experimental and clinical data suggest that intestinal microbiota may have an influence on the development of stroke risk factors, such as atherosclerosis. A stroke may result in a considerable change in the intestinal microbiome due to direct and indirect mechanisms. Experimental data indicate that the intestinal microbiota influences neuroinflammation after stroke by immunological mechanisms, and also contributes to systemic homeostasis. Which bacterial species have positive or negative modulating effects on a disease, such as has been described for cancer [60,61], has yet to be sufficiently investigated. The general problem of low reproducibility of results is particularly relevant in the field of microbiome research. It is therefore important to define and establish international methodological microbiome standards. Therefore, no specific recommendations for action can be determined for clinical practice, and in particular, modulation of intestinal microbiota cannot be recommended. This should even be discouraged, as the available data cannot rule out possible harmful effects. Consequently the only general recommendation is to pursue a healthy lifestyle, including good nutrition [47, 62]. However, the results so far suggest that new exciting and clinically-relevant discoveries in the field of microbiome - stroke research are to be expected in the coming years.

\section{Conflict of Interest}

No conflict of interest has been declared by the authors.

\section{References}

[1] Qin J, Li R, Raes J et al. A human gut microbial gene catalogue established by metagenomic sequencing. Nature 2010; 464: 59-65

[2] O'Hara AM, Shanahan F. The gut flora as a forgotten organ. EMBO Rep 2006; 7: 688-693

[3] Round JL, Mazmanian SK. The gut microbiota shapes intestinal immune responses during health and disease. Nat Rev Immunol 2009; 9: 313-323

[4] Braniste $\mathrm{V}, \mathrm{Al}$-Asmakh $\mathrm{M}$, Kowal $\mathrm{C}$ et al. The gut microbiota influences blood-brain barrier permeability in mice. Sci Transl Med 2014; 6: 263ra158 doi: 10.1126/scitranslmed.3009759

[5] Erny D, Hrabe de Angelis AL, Jaitin D et al. Host microbiota constantly control maturation and function of microglia in the CNS. Nat Neurosci 2015; 18: 965-977

[6] Hoban AE, Stilling RM, Ryan FJ et al. Regulation of prefrontal cortex myelination by the microbiota. Transl Psychiatry 2016; 6: e774

[7] Luczynski P, McVey Neufeld KA, Oriach CS et al. Growing up in a bubble: using germ-free animals to assess the influence of the gut microbiota on brain and behavior. Int J Neuropsychopharmacol 2016, doi:10.1093/ijnp/pyw020
[8] De Paepe M, Leclerc M, Tinsley CR et al. Bacteriophages: an underestimated role in human and animal health? Front Cell Infect Microbiol 2014; 4: 39

[9] Ogilvie LA, Jones BV. The human gut virome: a multifaceted majority. Frontiers in microbiology 2015; 6: 918

[10] Backhed F, Ley RE, Sonnenburg JL et al. Host-bacterial mutualism in the human intestine. Science 2005; 307: 1915-1920

[11] Nguyen TL, Vieira-Silva S, Liston A et al. How informative is the mouse for human gut microbiota research? Dis Model Mech 2015; 8: 1-16

[12] Houlden A, Goldrick M, Brough D et al. Brain injury induces specific changes in the caecal microbiota of mice via altered autonomic activity and mucoprotein production. Brain Behav Immun 2016, doi:10.1016/j.bbi.2016.04.003

[13] Singh V, Roth S, Llovera G et al. Microbiota dysbiosis controls the neuroinflammatory response after stroke. J Neurosci 2016; 36: 7428-7440

[14] Swidsinski AL-B V, Krüger M, Kirsch S. Central nervous system and the colonic bioreactor: analysis of colonic microbiota in patients with stroke unravels unknown mechanisms of the host defense after brain injury. Intest Res 2012; 10: 332-342

[15] Karlsson FH, Fak F, Nookaew I et al. Symptomatic atherosclerosis is associated with an altered gut metagenome. Nat Commun 2012; 3: 1245 doi: $10.1038 /$ ncomms 2266

[16] Koren O, Spor A, Felin J et al. Human oral, gut, and plaque microbiota in patients with atherosclerosis. Proc Natl Acad Sci USA 2010; 108: (Suppl 1): 4592-4598

[17] Yin J, Liao SX, He Y et al. Dysbiosis of gut microbiota with reduced trimethylamine- $\mathrm{N}$-oxide level in patients with large-artery atherosclerotic stroke or transient ischemic attack. J Am Heart Assoc 2015; 4: doi: 10.1161/JAHA.115.002699

[18] Mayer EA, Tillisch K, Gupta A. Gut/brain axis and the microbiota. J Clin Invest 2015; 125: 926-938

[19] Collins SM, Bercik P. The relationship between intestinal microbiota and the central nervous system in normal gastrointestinal function and disease. Gastroenterology 2009; 136: 2003-2014

[20] Schulte-Herbruggen O, Quarcoo D, Meisel A et al. Differential affection of intestinal immune cell populations after cerebral ischemia in mice. Neuroimmunomodulation 2009; 16: 213-219

[21] Caso JR, Hurtado O, Pereira MP et al. Colonic bacterial translocation as a possible factor in stress-worsening experimental stroke outcome. Am J Physiol Regul Integr Comp Physiol 2009; 296: R979-R985

[22] Tascilar N, Irkorucu O, Tascilar O et al. Bacterial translocation in experimental stroke: what happens to the gut barrier? Bratisl Lek Listy 2010; 111: 194-199

[23] Crapser J, Ritzel R, Verma R et al. Ischemic stroke induces gut permeability and enhances bacterial translocation leading to sepsis in aged mice. Aging (Albany NY) 2016; 8: 1049-1063

[24] Jandzinski M. Manipulation of the microbiome and its impact on functional recovery following ischemic stroke. Honors Scholar Theses Paper 414, 2015, http://digitalcommons.uconn.edu/srhonors_theses $/ 414$

[25] Stanley D, Mason LJ, Mackin KE et al. Translocation and dissemination of commensal bacteria in post-stroke infection. Nat Med 2016; 22: $1277-1284$

[26] Hannawi Y, Hannawi B, Rao CP et al. Stroke-associated pneumonia: major advances and obstacles. Cerebrovasc Dis 2013; 35: 430-443

[27] Meisel C, Schwab JM, Prass K et al. Central nervous system injury-induced immune deficiency syndrome. Nat Rev Neurosci 2005; 6: 775-786

[28] Prass K, Braun JS, Dirnagl U et al. Stroke propagates bacterial aspiration to pneumonia in a model of cerebral ischemia. Stroke 2006; 37: $2607-2612$ 
[29] Benakis C, Brea D, Caballero S et al. Commensal microbiota affects ischemic stroke outcome by regulating intestinal gammadelta T cells. Nat Med 2016; 22: 516-523

[30] Prass K, Meisel C, Hoflich C et al. Stroke-induced immunodeficiency promotes spontaneous bacterial infections and is mediated by sympathetic activation reversal by poststroke T helper cell type 1-like immunostimulation. J Exp Med 2003; 198: 725-736

[31] Dirnagl U, Klehmet J, Braun JS et al. Stroke-induced immunodepression: experimental evidence and clinical relevance. Stroke 2007; 38: 770-773

[32] Iadecola C, Anrather ]. The immunology of stroke: from mechanisms to translation. Nat Med 2011; 17: 796-808

[33] Chamorro A, Meisel A, Planas AM et al. The immunology of acute stroke. Nat Rev Neurol 2012; 8: 401-410

[34] Liesz A, Hu X, Kleinschnitz C et al. Functional role of regulatory lymphocytes in stroke: facts and controversies. Stroke 2015; 46: 1422-1430

[35] Chow J, Mazmanian SK. Getting the bugs out of the immune system: do bacterial microbiota "fix" intestinal T cell responses? Cell Host Microbe 2009; 5: 8-12

[36] Sun J, Wang F, Ling Z et al. Clostridium butyricum attenuates cerebral ischemia/reperfusion injury in diabetic mice via modulation of gut microbiota. Brain Res 2016; 1642: 180-188

[37] Winek K, Engel O, Koduah P et al. Depletion of cultivatable gut microbiota by broad-spectrum antibiotic pretreatment worsens outcome after murine stroke. Stroke 2016; 47: 1354-1363

[38] Nakasone C, Yamamoto N, Nakamatsu M et al. Accumulation of gamma/delta T cells in the lungs and their roles in neutrophil-mediated host defense against pneumococcal infection. Microbes Infect 2007; 9: 251-258

[39] Marsland BJ, Trompette A, Gollwitzer ES. The gut-lung axis in respiratory disease. Ann Am Thorac Soc 2015; 12: (Suppl 2): S150-S156

[40] Schuijt T], Lankelma JM, Scicluna BP et al. The gut microbiota plays a protective role in the host defence against pneumococcal pneumonia. Gut 2016; 65: 575-583

[41] Rosenfeld ME, Campbell LA. Pathogens and atherosclerosis: update on the potential contribution of multiple infectious organisms to the pathogenesis of atherosclerosis. Thromb Haemost 2011; 106: 858-867

[42] Wang Z, Klipfell E, Bennett B] et al. Gut flora metabolism of phosphatidylcholine promotes cardiovascular disease. Nature 2011; 472: 57-63

[43] Zhu W, Gregory JC, Org E et al. Gut microbial metabolite TMAO enhances platelet hyperreactivity and thrombosis Risk. Cell 2016; 165: 111-124

[44] Koeth RA, Wang Z, Levison BS et al. Intestinal microbiota metabolism of $\mathrm{L}$-carnitine, a nutrient in red meat, promotes atherosclerosis. Nat Med 2013; 19: 576-585

[45] Tang WH, Wang Z, Levison BS et al. Intestinal microbial metabolism of phosphatidylcholine and cardiovascular risk. N Engl J Med 2013; 368: 1575-1584

[46] Stepankova R, Tonar Z, Bartova J et al. Absence of microbiota (germ-free conditions) accelerates the atherosclerosis in ApoE-deficient mice fed standard low cholesterol diet. J Atheroscler Thromb 2010; 17: 796-804
[47] Singh V, Yeoh BS, Vijay-Kumar M. Gut microbiome as a novel cardiovascular therapeutic target. Curr Opin Pharmacol 2016; 27 : 8-12

[48] Troseid M, Ueland T, Hov JR et al. Microbiota-dependent metabolite trimethylamine- $\mathrm{N}$-oxide is associated with disease severity and survival of patients with chronic heart failure. J Intern Med 2015; 277: 717-726

[49] Troseid M, Hov JR, Nestvold TK et al. Major increase in microbiota-dependent proatherogenic metabolite TMAO one year after bariatric surgery. Metab Syndr Relat Disord 2016; 14: 197-201

[50] Sacco RL, Benjamin E], Broderick JP et al. American heart association prevention conference. IV. Prevention and rehabilitation of stroke. risk factors. Stroke 1997; 28: 1507-1517

[51] Winek K, Dirnagl U, Meisel A. The gut microbiome as therapeutic target in central nervous system diseases: implications for stroke. Neurotherapeutics 2016; 13: 762-774

[52] Winek K, Meisel A, Dirnagl U. Gut microbiota impact on stroke outcome: Fad or fact? J Cereb Blood Flow Metab 2016; 36: 891-898

[53] Schaller B], Graf R, Jacobs AH. Pathophysiological changes of the gastrointestinal tract in ischemic stroke. Am J Gastroenterol 2006; 101: 1655-1665

[54] Tottey W, Feria-Gervasio D, Gaci N et al. Colonic transit time is a driven force of the gut microbiota composition and metabolism: in vitro evidence. J Neurogastroenterol Motil 2017; 23: 124-134

[55] Jernberg C, Lofmark S, Edlund C et al. Long-term ecological impacts of antibiotic administration on the human intestinal microbiota. ISME J 2007; 1: 56-66

[56] Ravel ], Wommack KE. All hail reproducibility in microbiome research. 2014; DOI: D - NLM: PMC3975297 EDAT- 2014/03/08 06:00 MHDA- 2014/03/08 06:01 CRDT- 2014/03/08 06:00 PHST- 2014/03/03 [received] PHST- 2014/03/03 [accepted] AID - 2049-2618-2-8 [pii] AID - 10.1186/2049-2618-2-8 [doi] PST - epublish

[57] Servick K. Mouse microbes may make scientific studies harder to replicate. Science 2016, doi:10.1126/science.aah7199

[58] Bleich A, Hansen AK. Time to include the gut microbiota in the hygienic standardisation of laboratory rodents. Comp Immunol Microbiol Infect Dis 2012; 35: 81-92

[59] Sadler R, Singh V, Benakis C et al. Microbiota differences between commercial breeders impacts the post-stroke immune response. Brain Behav Immun 2017, doi:10.1016/j.bbi.2017.03.011

[60] Sivan A, Corrales L, Hubert $N$ et al. Commensal bifidobacterium promotes antitumor immunity and facilitates anti-PD-L1 efficacy. Science 2015; 350: 1084-1089

[61] Vetizou M, Pitt JM, Daillere R et al. Anticancer immunotherapy by CTLA-4 blockade relies on the gut microbiota. Science 2015; 350: 1079-1084

[62] Ferguson JF, Allayee H, Gerszten RE et al. Nutrigenomics, the microbiome, and gene-environment interactions: new directions in cardiovascular disease research, prevention, and treatment: a scientific statement from the american heart association. Circ Cardiovasc Genet 2016; 9: 291-313 